\title{
Factores incidentes en la construcción de confianza de los estudiantes hacia las autoridades escolares en la educación media superior en México
}

\author{
Factors involved in building trust among students towards \\ school authorities in upper secondary education media in \\ Mexico
}

DOI: $10.32870 /$ dse.v0i19.526

\section{Carlos Alberto Ramírez Sánchez*}

\begin{abstract}
Resumen
La confianza en las autoridades educativas por parte de los alumnos en la educación media superior (EMS) en México es un elemento fundamental para garantizar la calidad educativa, ya que su ausencia es un factor definitorio en el abandono (ENDEMS, 2012) y tiene graves afectaciones en el logro escolar (INEE, 2006). Por medio de un análisis de regresión logística, se averigua sobre los elementos que condicionan la confianza en las autoridades y qué peso tiene cada uno de estos factores. Se toma como referente al componente individual (o de composición estudiantil escolar), y el contextual (o de la gestión escolar) con el propósito de resaltar la importancia de la confianza en la elaboración de políticas públicas educativas en este nivel de estudios.
\end{abstract}

Palabras clave: confianza - educación - calidad educativa - abandono escolar - gestión escolar - composición escolar.

\begin{abstract}
Trust in the authorities by students in upper secondary education (EMS by its initials in Spanish) in Mexico is a fundamental element to guarantee educational quality, since the absence of this trust is a significant factor in student dropout (ENDEMS, 2012) and has serious effects on school achievement (INEE, 2006). Through a logistic regression analysis, this paper inquires into the elements that condition trust in the authorities and the relative weight each one of these factors has, taking as a reference the individual component (the school population) and the contextual one (the school management), in order to highlight the importance of trust in the development of public educational policies at this level of education.
\end{abstract}

Key words: trust - education - educational quality - school dropout - school management - school composition.

\footnotetext{
* Maestro en gobierno y asuntos públicos. Especialista en temas de política pública y acción gubernamental. Asistente de investigación en la
} Facultad Latinoamericana de Ciencias Sociales (FLACSO), sede México. México. carlos.ramirez@estudiante-flacso.mx 


\section{Introducción}

Dentro de la gestión educativa, referente a la organización y gobierno de la escuela, existe un factor exógeno que es determinante para que los alumnos se identifiquen con la visión y valores del sistema educativo y este es el clima escolar. A pesar de no ser un elemento que dependa directamente del centro educativo, es obligación de este establecer las condiciones necesarias para la sana convivencia entre alumnos y autoridades.

De manera cotidiana las autoridades escolares son percibidas por los alumnos como rivales a vencer, como parte de los obstáculos a superar para avanzar académicamente. La autoridad que ejerce poder de mando es percibida como la vencedora, la que impone sus reglas y visión del mundo a los vencidos, los alumnos. Es por eso que la brecha de comunicación y confianza entre estos dos actores tan relevantes para el sistema educativo se va haciendo cada vez más grande.

En los adolescentes actuales el respeto y la confianza hacia las autoridades escolares no están dados como algo natural e indiscutido, los profesores no tienen garantizada la obediencia, la escucha y el reconocimiento. Ello significa que el profesor no ostenta la autoridad que ha sido otorgada por la institución escolar [...]Los alumnos y los profesores tienen que cotidianamente construir la autoridad (Zamora, 2017: 4).

Se ha establecido la falta de confianza hacia las autoridades escolares, tomado como referentes a profesores, directivos y orientadores como factor importante para que un alumno abandone sus estudios de Educación Media Superior (EMS) (ENDEMS, 2012). El presente trabajo se fundamenta en las siguientes cuestiones: la primera es determinar ¿cuáles son los elementos condicionales para que exista confianza de los alumnos hacia las autoridades escolares y qué peso tiene cada uno de estos. La segunda es definir ¿cuál es la propensión marginal de los alumnos a confiar en las autoridades escolares, divididos en dos grupos, los que abandonaron sus estudios de EMS y los que concluyeron satisfactoriamente.

Precisando lo anterior, se puede hacer un análisis integral que ayude a dar una mejor lectura a las relaciones intraescolares, definidas como las interacciones cotidianas de los actores involucrados en el proceso educativo dentro de las escuelas de la EMS, principalmente las vinculadas a los alumnos y las autoridades escolares. Asimismo, con la propensión marginal a confiar, se podrá determinar si la falta de confianza es un factor importante a tomar en cuenta para estudiar las causas del abandono escolar, problema público de gran relevancia para el sistema educativo mexicano.

Para los propósitos del presente trabajo, los factores determinantes de la confianza en las autoridades escolares a tomar en cuenta se integran en dos dimensiones: las contextuales, que se dividen en características propias de la escuela, estructura y organización; y las individuales,

Diálo@os sobre Educación año 10 | número 19 | julio-diciembre 2019 | ISSN 2007-2171 
que tienen un efecto en el alumno de acuerdo con sus características propias; ejemplos de esto son la composición y características del alumnado, así como las variables socioeconómicas.

En el marco de las observaciones anteriores, este trabajo parte de las siguientes hipótesis: 1) los factores contextuales tienen un mayor peso en la generación de confianza de la autoridad escolar hacia sus alumnos, y 2) Existe una mayor propensión marginal a confiar en las autoridades por parte de los alumnos no abandonantes que de los abandonantes.

Sobre la base de lo anteriormente planteado, se fundamenta la importancia de realizar un análisis acerca de los mecanismos generadores de confianza de los alumnos hacia las autoridades escolares, ya que establecer cuáles son los factores que inciden en propiciarla son determinantes para la creación de políticas públicas enfocadas en el mejoramiento de las relaciones intraescolares y la calidad educativa.

Para este caso, se decidió tomar a la educación media superior ya que, según la Encuesta Nacional de Deserción Escolar en la Educación Media Superior (ENDEMS, 2012), la edad típica de estudio de este nivel educativo es de los 15 a los 17 años, edad en la que los adolescentes experimentan sus primeras veces: "la primera relación sexual, el primer trabajo, la primera salida de vivir en el hogar paterno, el primer embarazo, etcétera; todas ellas se producen, mayoritariamente, entre los 15 y 19 años" (Pérez, 2017: 12). De esta manera, para los adolescentes, la escuela es un espacio medular para la formación de una experiencia estudiantil aún más allá de lo enseñado de manera formal por el Sistema Educativo Nacional (SEN).

De acuerdo con el Instituto Nacional para la Evaluación de la Educación (INEE, 2010), la autoridad escolar dentro de los centros educativos debe cumplir con las siguientes habilidades y funciones: 1) visión clara de las escuelas, sus deficiencias y su potencial, 2) capacidad de implementación de métodos que faciliten el aprendizaje de los alumnos, 3) promover un ambiente de trabajo ordenado y seguro, 4) incentivar un clima de aprendizaje académico, 5) facilitar la integración de los padres de familia en los asuntos del plantel. Dadas las características anteriores, para el presente trabajo se tomarán como autoridades escolares a los directivos, maestros y prefectos, ya que son los actores involucrados directamente con las labores planteadas.

El presente trabajo está dividido en cuatro partes. En la primera se realiza un análisis teórico-conceptual de la confianza en los centros educativos, así como de la gestión y la composición escolar como elementos clave de la generación de esta confianza dentro de la EMS. En el segundo apartado se hace una descripción de la metodología aplicada, así como la fuente de información empleada. En una tercera sección se plantea y discute el modelo, así como las variables empleadas. Finalmente, en el cuarto apartado se hace un análisis de los resultados y se estudian sus posibles repercusiones en materia de política educativa. 


\section{Confianza educativa}

De acuerdo con Cornejo et al. (2010), la confianza es la relación dada entre dos o más personas, donde, a pesar de la vulnerabilidad de alguna de las partes, no existe sometimiento o coerción, de forma que confiar en alguien corresponde a una disposición positiva respecto de las intenciones o comportamientos de otro u otra (Mayer et al., 1995).

Autores como Kramer (1999) y Abarca (2004) explican que la confianza se da por lo general en condiciones asimétricas de poder, en las que, ante la incertidumbre de las intenciones de uno de los grupos sobre el otro, se decide tomar parte en la misma dirección que el individuo o individuos de un nivel jerárquico superior. Para que haya confianza debe haber riesgo y un mínimo de incertidumbre, así como una interdependencia de los fines, es decir que para que los objetivos del individuo o grupo más vulnerable puedan alcanzarse, necesitan la cooperación de su contraparte.

De esta manera, la confianza no es un término estático sino un proceso dinámico, en el que distintos factores pueden hacer que la confianza entre dos grupos pueda aparecer y desaparecer de manera súbita. Es importante no confundir el término de confianza con el de sumisión, ya que, si bien ambos estímulos están orientados al seguimiento de determinadas pautas conductuales, el primero se realiza de una manera libre y consensuada, mientras que en el segundo existe una amenaza coercitiva que genera un resentimiento latente de una de las partes, que puede terminar por afectar la dinámica en ciertas condiciones que pongan en duda la supremacía jerárquica del grupo o persona dominante.

Bryk y Schneider (2003) definen la confianza relacional como aquella dada entre roles sociales diferenciados, dentro de una organización con distintos grados jerárquicos delimitados, de tal forma que cada una de las partes involucradas está consciente de las obligaciones que posee su rol y al mismo tiempo mantiene ciertas expectativas acerca de las obligaciones de los otros grupos. De esta manera, para que un centro educativo funcione bien, tanto los alumnos como las autoridades escolares, deben estar conscientes de sus derechos y obligaciones, así como la responsabilidad que tiene con las otras partes debido a la interdependencia de objetivos.

La importancia de que exista una relación de confianza en el sistema educativo es tener la oportunidad de crear un canal simétrico de comunicación, donde, si bien tanto los alumnos como las autoridades escolares poseen distintos roles dentro de la estructura organizacional de la escuela, se puede abrir un diálogo entre ambos grupos. De esta manera, las autoridades escolares pueden conocer más a fondo las necesidades, inquietudes y problemas de los alumnos; esto con el propósito de tener una retroalimentación que ayude a la mejora de las estrategias educativas y de gestión escolar.

La confianza en las autoridades escolares es una parte integral de la educación de calidad, término derivado de las reformas constitucionales de 2013 en las que se incorpora la figura de educación de calidad al artículo $3^{\circ}$, en la que se presenta al Estado como máximo garante y

Diólo@os año 10 | número 19 | julio-diciembre 2019 | ISSN 2007-2171 
responsable del logro educativo, y al artículo $8^{\circ}$ de la Ley General de Educación (LGE) en la cual se define la calidad educativa como "la congruencia entre los objetivos, resultados y procesos del SEN, conforme a las dimensiones de eficacia, eficiencia, pertinencia y equidad" (LGE, 2013). El término de calidad educativa funge como principal directriz del Nuevo Modelo Educativo (2013), en el que se resaltan los términos de eficacia, eficiencia, pertinencia y equidad, en donde el Estado tiene la obligación de equilibrar las condiciones iniciales garantizando así la permanencia y el logro educativo.

De acuerdo con la UNESCO (1992), la calidad de la educación supone garantizar desde el Estado la formulación de políticas que posibiliten la igualdad de acceso, la permanencia y egreso de la población a los sistemas educativos en el marco de la equidad social, otra propuesta humana y de orden internacional. Es así que la confianza es un indicador clave de la calidad educativa, que permite obtener retroalimentación de las políticas públicas ejercidas mediante una relación de cercanía entre alumnos y autoridades escolares.

\section{Factores clave en la generación de confianza}

La formulación de una acertada política pública comienza a construirse desde su diseño, el cual tiene como base la definición del problema, por lo que deben conocerse de manera precisa las características de los involucrados, tanto de parte de la oferta (gestión e infraestructura educativa) como de la demanda (composición estudiantil).

Si bien es cierto que la generación de confianza es un proceso dinámico y multicausal (Bryk y Schneider, 2003), se pueden agrupar las variables en dos grandes factores que faciliten su análisis y su estudio, la dimensión contextual referente a aquellos aspectos exógenos al alumno y determinados por el entorno, y la dimensión individual, concerniente a las características propias de los estudiantes, que no están ligadas de manera directa a la gestión escolar ni al gobierno de la escuela.

\section{La gestión escolar como fundamento de la dimensión contextual}

La escuela, como institución, es responsable de crear y promover las condiciones de aprendizaje y desarrollo humano, fundamentales en la concepción de educación de calidad, de manera que se fortalezcan los objetivos institucionales y los estudiantes se sientan pertenecientes y afines a los valores del SEN. El propósito de este orden escolar es disminuir los potenciales problemas pedagógicos, disciplinarios y de abandono que se pudieran generar dentro de las escuelas.

Esta organización interna de las escuelas es llamada gestión escolar, y es definida como "el proceso que - directamente vinculado con el gobierno de la escuela - da lugar a una serie de reflexiones y acciones de los actores, relacionadas con la formación de los alumnos y con los objetivos institucionales" (Barrientos Noriega et al., 2008: 3).

Si bien el término de gestión escolar hace referencia al gobierno de la escuela de una manera introspectiva, en él también entran factores relacionados con la interacción cotidiana de 
los actores directos de la acción educativa en las escuelas como: maestros, directivos, prefectos, orientadores y alumnos. Además, es importante mencionar que desde la instauración de la reforma educativa en 2013 se buscó dotar de más autonomía a cada uno de los centros educativos; sin embargo, tal libertad solamente deslindó a la federación de ciertos aspectos administrativos y logísticos (involucrando más a los padres de familia), pero en aspectos de gestión y operación de elementos esenciales a las necesidades de las escuelas, quedaron supeditados a lo dictado por un organismo central.

De esta manera, la función del personal escolar involucrado con la gestión de la escuela (directivos, profesores, orientadores y prefecto) se enfoca a la contención o sanción de problemas disciplinarios. Ante la situación planteada, la relación constitutiva del centro educativo, donde la relación docente-alumno es integral para la identificación del alumno con los valores del SEN, se crea un clima escolar asimétrico en el que hay una pérdida de confianza en el personal involucrado con la gestión educativa.

Por lo que se refiere el clima escolar, las escuelas que se organizan de forma efectiva funcionan de manera adecuada y logran efectos significativos, tanto en la mejora de la calidad educativa como en la retención y eficiencia terminal de los estudiantes.

Una vez cubierta una dotación mínima de recursos, ya no son los recursos disponibles, sino los procesos psicosociales y las normas que caracterizan las interacciones que se desarrollan en la institución escolar (considerada como un sistema social dinámico, con una cultura propia) lo que realmente diferencia a unas de otras, en su configuración y en los efectos obtenidos en el aprendizaje y permanencia (Cornejo at al., 2001: 12).

El logro educativo, la permanencia y la eficiencia terminal, no dependen únicamente de las características interpersonales del alumno o del personal involucrado con la gestión escolar, sino en buena medida de las interacciones y los espacios intersubjetivos, es decir, el contexto donde se dan estas relaciones, definido por Cornejo (2001) como clima escolar. Estos factores interpersonales se expresan en tres niveles:

- Institucional. Normas de convivencia y gestión educativa.

- Aula. Clima del aula. Relaciones docente-alumno y alumno-alumno.

- Intrapersonal. Auto concepto, creencias y valores de estudiantes y docentes.

En resumen, el clima escolar de una escuela es la "percepción que tienen los sujetos acerca de las relaciones interpersonales que establecen en el contexto escolar (a nivel de aula o de centro) y el contexto o marco en el cual estas interacciones se dan" (Cornejo et al., 2001: 15). 
Una distinción importante es que el clima escolar puede ser estudiado desde el enfoque de la gestión educativa o desde los procesos interpersonales que ocurren dentro de la institución. Asimismo, en este trabajo se definen las variables contextuales como aquellas pertenecientes a la gestión escolar, como gobierno de la escuela y del SEN.

El clima escolar es un factor determinante en la generación de confianza educativa (Bryk y Schneider, 2003), y un clima de aprendizaje positivo propicia una educación de calidad (Cornejo at al., 2010). La relevancia de este componente dentro de esta investigación es que depende en gran medida de la gestión escolar y el gobierno de la escuela, razón por la cual se puede tratar, tanto de manera integral como focalizada.

\section{Composición escolar}

El contexto escolar, en específico la composición de sus integrantes, tiene un alto impacto en el logro educativo, en la permanencia y la eficiencia terminal. Según Kreft (1993) el logro y la calidad educativa son el resultado de la composición del alumnado en la escuela, producto de la selectividad de esta, de tal manera que "las actitudes y los comportamientos individuales de los estudiantes dependen del tipo de alumno atraído o seleccionado por la escuela, lo cual moldea su composición y determina el clima escolar" (Cervini Iturre, 2003).

Es así que métricas como el logro educativo, la permanencia y la eficiencia terminal, dependen en gran medida de la composición estudiantil, de tal forma que toda investigación sobre comportamiento escolar debe de tener en cuenta la composición escolar, la cual está determinada por los siguientes factores:

- Sociodemográficos. Los cuales incluyen origen social, etnia, sexo, capital cultural y logros anteriores.

- Socioeconómicos. Son aquellos que tienen que ver con el ingreso y poder adquisitivo del alumno y de su familia, así como del gasto que se dedica a temas educativos.

De esta manera, según lo planteado por Kreft (1993), el tipo de escuela, y por tanto la población atraída, determinará el clima escolar y, en consecuencia, tendrá un efecto sobre las normas, el comportamiento y el rendimiento individual de los estudiantes, lo que repercutirá en el logro escolar, la permanencia y la eficiencia terminal. Una de las hipótesis manejadas por este autor es que "como resultado de la selectividad, las poblaciones estudiantiles en las escuelas privadas serán diferentes a las de las escuelas públicas" (Kreft, 1993).

En este trabajo se toman en cuenta variables de composición escolar debido a que las características de la demanda educativa tienen un efecto importante en las decisiones de abandono escolar. Dentro del esquema planteado para esta investigación, que se desarrolla en el siguiente apartado, estos factores entran en la dimensión individual debido a que no tienen 
una relación directa con la gestión escolar, sino que son particularidades de las poblaciones específicas.

Las características propias del alumnado pueden crear una predisposición a la generación de confianza en las autoridades o, en contraparte, pueden propiciar un distanciamiento aún mayor, lo cual puede afectar negativamente la dinámica dentro del centro de estudio. Las variables que integran esta categoría permitirán identificar aquellas características de los alumnos que impiden un estrechamiento de los vínculos con las autoridades escolares, para así poder ser tratadas tanto de manera focalizada como integral.

Tanto la gestión como la composición escolar son factores que, si bien en este trabajo son analizados por separado para determinar el peso de cada uno en la generación de confianza, no son mutuamente excluyentes, por lo tanto, es necesario realizar un análisis integral de todas las piezas.

\section{Fuente de información y metodología}

La fuente de información utilizada es la Encuesta Nacional de Deserción Escolar en la Educación Media Superior (ENDEMS) publicada en 2012. ${ }^{1}$ Es una encuesta de corte transversal de tipo simple, con entrevistas personales cara a cara, sin ningún tipo de incentivos a los entrevistados y representativa en el nivel nacional.

Para esta encuesta, las viviendas componen la unidad de muestreo, donde esta última es definida como el espacio delimitado por paredes y techos de cualquier material de construcción, donde viven, duermen, preparan alimentos, comen y se protegen de las inclemencias del tiempo una o más personas. De igual manera, las viviendas deben tener entrada independiente.

Por otra parte, la unidad de observación es conformada por el hogar, el cual comprende a todas las personas que comparten la misma vivienda y se sostienen de un gasto común. Cabe resaltar que una persona que vive sola también constituye un hogar.

La población objetivo de esta encuesta está constituida por hombres y mujeres entre los 14 y 25 años cumplidos, que residen permanentemente en viviendas particulares ubicadas en localidades con más de 500 habitantes. Asimismo, el periodo de levantamiento de la información fue del 25 de junio al 30 de julio de 2011 cuyo esquema de muestreo fue probabilista, estratificado por conglomerados.

\section{Modelo de probabilidad: Logit}

Existen cierto tipo de variables dependientes que tienen un carácter limitado, es decir, forman parte de un modelo de regresión cuya respuesta es de carácter cualitativo, mientras que las variables independientes pueden ser de naturaleza cualitativa o cuantitativa. Por lo general, la

1 Es importante resaltar que esta encuesta perdió continuidad, por lo cual su última publicación fue para el año 2012 y no se ha retomado hasta la fecha. 
variable de respuesta se restringe a dos valores — se pueden presentar casos donde pueden categorizarse en tres (tricotómica) o más términos (polinómica). Existen distintos modelos de probabilidad para el análisis de una variable de respuesta binaria:

- El modelo lineal de probabilidad (MLP).

- El modelo logit.

- El modelo probit.

- El modelo tobit.

Para los propósitos de este modelo, estimar el peso de cada una de las variables seleccionadas en la variación de la probabilidad de tener o no confianza en las autoridades escolares, el modelo que mejor se ajusta a las condiciones de las variables es el modelo logit, debido a que cumple con las siguientes ventajas respecto a las otras alternativas: 1) las probabilidades calculadas siempre están entre 0 y 1-2, descarta las probabilidades negativas, 3) no asume que el término del error esté distribuido normalmente.

Entre los modelos logit el término del error toma una distribución logística. La función de densidad de probabilidad (FDP) es la probabilidad relativa de que una variable aleatoria tome determinado valor, y para el caso de una variable aleatoria distribuida logísticamente se presenta de la siguiente manera:

$$
L(X)=\frac{e^{-x}}{\left(1+e^{-x}\right)^{2}} \quad \text { Ecuación 1 }
$$

Así pues, la probabilidad de que un término de error $\epsilon$ distribuido logísticamente sea menor que $\mathrm{X}$ está dado por:

$$
\operatorname{Pr}(€<X)=\frac{1}{1+e^{-X}} \quad \text { Ecuación } 2
$$

Como ya se definió anteriormente, el modelo logit supone una variable dicotómica dependiente $Y$ que se igual a 1 cuando una variable latente $Y^{*}>0$, en la cual:

$$
Y i^{*}=\beta_{0}+\beta_{1} x_{1} i+\ldots+\beta_{k} x_{k i}+\varepsilon_{i} \quad \text { Ecuación } 3
$$


Donde $\varepsilon_{i}$ toma una distribución logística. Por tanto, usando la ecuación 10 se puede reescribir de la siguiente manera:

$$
\begin{array}{cc}
\log L(\beta)=\sum_{Y=1} \log \left(1-\frac{1}{1+e^{-} \beta_{0}+\beta_{1} x_{1} i+\ldots+\beta_{k} x_{k i}}\right) & \text { Ecuación } 4 \\
\sum_{Y=0} \log \left(1-\frac{1}{1+e^{-} \beta_{0}+\beta_{1} x_{1} i+\ldots+\beta_{k} x_{k i}}\right) & \text { Ecuación } 5
\end{array}
$$

De esta manera se seleccionan como estimados los valores de los parámetros $\beta$ que maximicen la función logarítmica de verosimilitud.

Con base en lo anteriormente expuesto, se utiliza este enfoque analítico debido a que el objetivo de este apartado es establecer el peso de los factores (contextuales e individuales) en la confianza que un alumno de EMS tiene en sus autoridades (profesores, directivos y orientadores), esto conforme a la información disponible en la ENDEMS (2012).

\section{Diseño del modelo y selección de variables}

Dadas las consideraciones anteriores, el modelo adopta la estructura de la ecuación 3, donde Yi ${ }^{*}$ es una variable dicotómica que solamente toma dos valores: 1 (Tiene confianza) y 0 (No tiene confianza). $\beta_{0}$ es el intercepto que representa la proporción de estudiantes que tienen confianza en las autoridades manteniendo constantes todas las variables. $\beta_{1}$ es el momio asociado a una variable categórica manteniendo todas las demás variables constantes, $X^{i}{ }_{1}$ es una variable independiente y predictora de la variable dependiente y es el error o la varianza no explicada en el modelo.

Para el presente modelo logístico se trabaja con variables categóricas debido a que la fuente de información (ENDEMS, 2012) se encuentra en este formato, además, la interpretación es más sencilla ya que se puede analizar un cambio en la variable dependiente a través de las subcategorías de las variables explicativas.

Un aspecto importante por considerar es que para la construcción de la base de datos se toma únicamente en consideración a los alumnos que estuvieron matriculados alguna vez en la EMS (abandonantes y egresados), dejando fuera a los entrevistados que nunca estuvieron inscritos, esto debido a que el propósito de este artículo es analizar la confianza de los entrevistados que interactuaron alguna vez con autoridades escolares.

Para la selección de variables se consideraron dos criterios: 1) que pudieran integrarse en uno de los dos factores analizados, ya sean los de carácter contextual o individual, y 2) que estuvieran presentes en la fuente de información, la ENDEMS (2012). Cabe resaltar que, por ser una 
encuesta enfocada a los estudiantes, la mayoría de las preguntas están orientadas al individuo, aunque sí hay elementos suficientes para hacer una categorización contextual.

Es así como mediante este modelo se puede ver el peso individual de cada variable explicativa en la variable independiente, que en este caso es la confianza de los alumnos de la EMS en sus autoridades escolares (profesores, orientadores y directivos). De esta manera se puede analizar de forma estructurada cada uno de los factores que inciden en este aspecto tan importante para la gestión y el clima escolar que es la cercanía y confianza de un joven con el gobierno de la escuela. A continuación, se realiza una descripción de las variables utilizadas.

\begin{tabular}{|c|c|c|}
\hline \multicolumn{3}{|c|}{ Descripción de las variables dependientes } \\
\hline Categoría & Descripción de la variable & Variable \\
\hline $\begin{array}{l}\text { Institución } \\
\text { educativa }\end{array}$ & Variable dicotómica & $\begin{array}{l}\text { Se estudió la EMS en un CO- } \\
\text { NALEP (Sí/No) }\end{array}$ \\
\hline \multirow[t]{8}{*}{ Contextual } & Variable dicotómica & $\begin{array}{l}\text { Se estudió la EMS en un CETIS } \\
\text { (Sí/No) }\end{array}$ \\
\hline & Variable dicotómica & $\begin{array}{l}\text { Se estudió la EMS en un Bachille- } \\
\text { rato Estatal (Sí/No) }\end{array}$ \\
\hline & Variable dicotómica & $\begin{array}{l}\text { Se estudió la EMS en una prepa- } \\
\text { ratoria abierta (Sí/No) }\end{array}$ \\
\hline & Variable dicotómica & $\begin{array}{l}\text { Se estudió la EMS en una prepa- } \\
\text { ratoria privada (Sí/No) }\end{array}$ \\
\hline & Variable dicotómica & $\begin{array}{l}\text { Se estudió la EMS en un CECyT } \\
\text { (Si/No) }\end{array}$ \\
\hline & Variable dicotómica & $\begin{array}{l}\text { Se estudió la EMS en un Teleba- } \\
\text { chillerato (Sí/No) }\end{array}$ \\
\hline & Variable dicotómica & $\begin{array}{l}\text { Se estudió la EMS en una prepa- } \\
\text { ratoria autónoma (Sí/No) }\end{array}$ \\
\hline & Variable dicotómica & $\begin{array}{l}\text { Se estudió la EMS en un Bachille- } \\
\text { res (Sí/No) }\end{array}$ \\
\hline $\begin{array}{l}\text { Tipo de Bachi- } \\
\text { llerato }\end{array}$ & $\begin{array}{l}\text { Variable categórica. Tipo de bachillerato } \\
\text { donde se estudió }\end{array}$ & Bachillerato General \\
\hline \multirow{2}{*}{\multicolumn{2}{|c|}{ Contextual }} & Bachillerato Tecnológico \\
\hline & & Carrera Técnica \\
\hline Sostenimiento & $\begin{array}{l}\text { Variable categórica. Tipo de sostenimiento } \\
\text { del bachillerato donde se estudió. }\end{array}$ & Privado \\
\hline \multirow[t]{2}{*}{ Contextual } & & Público \\
\hline & & Mixto \\
\hline Con quién vivía & $\begin{array}{l}\text { Variable categórica. Con quién se vivía al } \\
\text { momento de estudiar la EMS }\end{array}$ & Otro \\
\hline \multirow{2}{*}{\multicolumn{2}{|c|}{ Individual }} & Mamá \\
\hline & & Papá \\
\hline
\end{tabular}




\begin{tabular}{|c|c|c|}
\hline $\begin{array}{l}\text { Cuando había } \\
\text { problemas }\end{array}$ & $\begin{array}{l}\text { Variable categórica. Cuando se tenía un pro- } \\
\text { blema referente a la escuela, con quién se } \\
\text { acudía }\end{array}$ & Acudía a papá \\
\hline \multirow{3}{*}{ Individual } & & Acudía a mamá \\
\hline & & Acudía a amigo \\
\hline & & Acudía a hermanos \\
\hline $\begin{array}{l}\text { Cómo percibía } \\
\text { su promedio }\end{array}$ & $\begin{array}{l}\text { Variable categórica. Cuál es la percepción del } \\
\text { promedio durante los estudios de EMS }\end{array}$ & Alto \\
\hline \multirow{3}{*}{ Individual } & & Regular \\
\hline & & Bajo \\
\hline & & Muy bajo \\
\hline Beca & \multirow[t]{2}{*}{ Variable dicotómica } & \multirow{2}{*}{$\begin{array}{l}\text { Al momento de estudiar la EMS } \\
\text { se contaba con beca (Sí/No) }\end{array}$} \\
\hline Contextual & & \\
\hline $\begin{array}{l}\text { Problemas } \\
\text { durante el ba- } \\
\text { chillerato }\end{array}$ & Variable dicotómica & $\begin{array}{l}\text { Al momento de estudiar la EMS: } \\
\text { te faltaba dinero (Sí/No) }\end{array}$ \\
\hline \multirow[t]{6}{*}{ Contextual } & Variable dicotómica & $\begin{array}{l}\text { Al momento de estudiar la EMS: } \\
\text { reprobaste materias (Sí/No) }\end{array}$ \\
\hline & Variable dicotómica & $\begin{array}{l}\text { Al momento de estudiar la EMS: } \\
\text { te disgustaba estudiar (Sí/No) }\end{array}$ \\
\hline & Variable dicotómica & $\begin{array}{l}\text { Al momento de estudiar la EMS: } \\
\text { tuviste problemas con las reglas } \\
\text { (Sí/No) }\end{array}$ \\
\hline & Variable dicotómica & $\begin{array}{l}\text { Al momento de estudiar la EMS: } \\
\text { sufriste discriminación (Sí/No) }\end{array}$ \\
\hline & Variable dicotómica & $\begin{array}{l}\text { Al momento de estudiar la EMS: } \\
\text { Embarazaste o quedaste emba- } \\
\text { razada (Sí/No) }\end{array}$ \\
\hline & Variable dicotómica & $\begin{array}{l}\text { Al momento de estudiar la EMS: } \\
\text { tuviste problemas con las insta- } \\
\text { laciones (Sí/No) }\end{array}$ \\
\hline Trabajo & Variable dicotómica & $\begin{array}{l}\text { Al momento de estudiar la EMS } \\
\text { trabajabas (Sí/No) }\end{array}$ \\
\hline \multicolumn{3}{|l|}{ Individual } \\
\hline $\begin{array}{l}\text { Nivel aspira- } \\
\text { cional }\end{array}$ & $\begin{array}{l}\text { Variable categórica. Al momento de estudiar } \\
\text { la EMS cual era el nivel académico al que as- } \\
\text { pirabas }\end{array}$ & Superior \\
\hline \multirow[t]{2}{*}{ Individual } & & Posgrado \\
\hline & & Otro \\
\hline $\begin{array}{l}\text { Uso de sustan- } \\
\text { cias }\end{array}$ & Variable dicotómica & $\begin{array}{l}\text { Al momento de estudiar la EMS } \\
\text { consumías drogas (Sí/No) }\end{array}$ \\
\hline
\end{tabular}


Factores incidentes en la construcción de confianza de los estudiantes hacia las autoridades escolares en la educación media superior en México

\begin{tabular}{|c|c|c|}
\hline Individual & Variable dicotómica & $\begin{array}{l}\text { Al momento de estudiar la EMS } \\
\text { consumías alcohol y/o tabaco } \\
\text { (Sí/No) }\end{array}$ \\
\hline Promedio & $\begin{array}{l}\text { Variable categórica. En términos numéricos } \\
\text { cuál fue el promedio en la EMS }\end{array}$ & 6 o más \\
\hline \multirow[t]{3}{*}{ Contextual } & & 70 más \\
\hline & & 8 o más \\
\hline & & 90 más \\
\hline Asistencia & $\begin{array}{l}\text { Variable categórica. Qué tanto se asistía a } \\
\text { clases }\end{array}$ & Regularmente \\
\hline \multirow[t]{2}{*}{ Individual } & & Faltaba mucho \\
\hline & & Casi no iba \\
\hline Edad & Variable categórica. Rango de edad & En rango \\
\hline Individual & & Fuera de rango \\
\hline
\end{tabular}




\section{Resultados}

\begin{tabular}{|c|c|c|c|c|c|}
\hline \multicolumn{5}{|c|}{ Confianza en las autoridades escolares } & \multirow[b]{2}{*}{$\mathrm{P}>\mathrm{Z}$} \\
\hline Categoría & Variable & $\mathrm{dy} / \mathrm{dx}$ & Std. Err. & $\mathrm{z}$ & \\
\hline \multirow[t]{9}{*}{ Institución educativa } & CONALEP & -0.059308 & 0.0252 & -2.35 & $0.019 * *$ \\
\hline & CETIS & -0.037485 & 0.0223 & -1.68 & $0.093^{*}$ \\
\hline & Bachillerato Estatal & -0.002775 & 0.0246 & -0.11 & 0.91 \\
\hline & Preparatoria Abierta & 0.121919 & 0.0395 & 3.09 & $0.002^{* * * *}$ \\
\hline & Preparatoria Privada & 0.0486851 & 0.0296 & 1.64 & $0.1 *$ \\
\hline & CECyT & 0.0121938 & 0.0353 & 0.35 & 0.73 \\
\hline & Telebachillerato & -0.0150379 & 0.0592 & -0.25 & 0.8 \\
\hline & Preparatoria Autónoma & 0.0296622 & 0.0236 & 1.26 & 0.209 \\
\hline & Bachilleres & 0.0244355 & 0.0225 & 1.08 & 0.278 \\
\hline \multirow[t]{2}{*}{ Tipo de Bachilerato } & Bach Tec & 0.0525683 & 0.0145 & 3.61 & $0 * * *$ \\
\hline & Carrera Tec & 0.0705931 & 0.0237 & 2.98 & $0.003^{* * * *}$ \\
\hline \multirow[t]{2}{*}{ Sostenimiento } & Privado & 0.0483003 & 0.0279 & 1.73 & $0.084 *$ \\
\hline & Mixto & -0.0269871 & 0.14 & -0.19 & 0.847 \\
\hline \multirow[t]{2}{*}{ Con quién vivía } & Mamá & -0.0502483 & 0.029 & -1.73 & $0.083^{*}$ \\
\hline & Papá & -0.0534165 & 0.0198 & -2.69 & $0.007 * * *$ \\
\hline \multirow[t]{4}{*}{ Cuando había problemas } & Acudia a papá & -0.0573413 & 0.00658 & -8.72 & $\mathrm{O}^{* * * *}$ \\
\hline & Acudia a mamá & -0.0413237 & 0.00763 & -5.41 & $0 * * *$ \\
\hline & Acudia a amigo & -0.0565273 & 0.0058 & -9.75 & $0 * * *$ \\
\hline & Acudia a hermanos & -0.046771 & 0.00569 & -8.22 & $0^{* * * *}$ \\
\hline \multirow[t]{4}{*}{ Cómo percibía su promedio } & Alto & -0.050641 & 0.0235 & -2.16 & $0.031 * *$ \\
\hline & regular & -0.078541 & 0.0247 & -3.18 & $0.001^{* * *}$ \\
\hline & Bajo & -0.044233 & 0.0286 & -1.55 & 0.122 \\
\hline & Muy bajo & 0.024018 & 0.0594 & 0.4 & 0.686 \\
\hline Beca & Tenía beca & 0.0461266 & 0.0152 & 3.04 & $0.002 * * *$ \\
\hline \multirow{7}{*}{ Problemas durante el bachillerato } & Falta de dinero & 0.0180869 & 0.0119 & 1.51 & 0.13 \\
\hline & Reprobar & 0.0440681 & 0.0261 & 1.69 & $0.091^{*}$ \\
\hline & Disgustaba estudiar & -0.0396787 & 0.0187 & -2.13 & $0.033 * *$ \\
\hline & Reglas & 0.0284257 & 0.0155 & 1.83 & $0.067 *$ \\
\hline & Discriminación & -0.0264881 & 0.0244 & -1.09 & 0.277 \\
\hline & Embarazo & 0.0369006 & 0.0255 & 1.45 & 0.147 \\
\hline & Instalaciones & -0.0478143 & 0.0205 & -2.33 & $0.02 * *$ \\
\hline Tabajo & Trabajaba & 0.042706 & 0.0144 & 2.97 & $0.003 * * *$ \\
\hline \multirow[t]{3}{*}{ Nivel aspiracional } & Superior & 0.0343971 & 0.0169 & 2.03 & $0.042 * *$ \\
\hline & Posgrado & 0.0594949 & 0.0193 & 3.08 & $0.002 * *$ \\
\hline & Otro & -0.0491292 & 0.0422 & -1.16 & 0.245 \\
\hline \multirow[t]{2}{*}{ Uso de sustancias } & Drogas & 0.0425384 & 0.0361 & 1.18 & 0.239 \\
\hline & Alcohol y/o Tabaco & -0.0264078 & 0.0128 & -2.07 & $0.039 * *$ \\
\hline \multirow[t]{4}{*}{ Promedio } & 6 o más & 0.0034799 & 0.0391 & 0.09 & 0.929 \\
\hline & 7 o más & 0.0510908 & 0.0202 & 2.53 & $0.011 * *$ \\
\hline & 8 o más & 0.034672 & 0.0189 & 1.84 & $0.066^{*}$ \\
\hline & 9 o más & 0.0637992 & 0.0246 & 2.59 & $0.01 * * *$ \\
\hline \multirow[t]{3}{*}{ Asistencia } & Regularmente & -0.0373486 & 0.0132 & -2.84 & $0.005^{* * * *}$ \\
\hline & Faltaba mucho & -0.0025212 & 0.0292 & -0.09 & 0.931 \\
\hline & Casi no iba & -0.0069554 & 0.0648 & -0.11 & 0.914 \\
\hline \multirow[t]{2}{*}{ Edad } & En rango & -0.0023995 & 0.013 & -0.18 & 0.853 \\
\hline & Muy grande & -0.0054868 & 0.0174 & -0.32 & 0.753 \\
\hline
\end{tabular}

Diólo Oos sobre Educación año 10 | número 19 | julio-diciembre 2019 | ISSN 2007-2171 


\section{Discusión de los resultados y conclusiones}

Por medio del modelo de regresión logística anterior se pudo estimar qué factores tienen influencia, ya sea positiva o negativa, en la confianza que los alumnos de EMS tienen en las autoridades escolares, ya sean maestros, prefectos o directivos. Es importante recordar que estos resultados están delimitados a la información dada por la Encuesta Nacional de Deserción en la Educación Media Superior publicada en 2012.

La estimación del modelo de regresión logística fue diseñada bajo el concepto de parsimonia, es decir, que con la menor cantidad de variables posibles se explicara la mayor proporción de varianza, de tal manera que las variables seleccionadas fueron aquellas que tuvieran cabida en las dos grandes dimensiones conceptuales abordadas en este trabajo: la contextual y la individual.

En cuanto al ajuste del modelo la pseudo $\mathrm{r}^{2}$, un indicador de la bondad de ajuste del modelo muestra un nivel de 0.1875 , además de esto, se realizaron las pruebas BIC (criterio de información bayesiana), el cual es una aproximación a una transformación de la probabilidad posterior de un modelo y AIC (Criterio de Información de Akaike), un estimador insesgado asintótico de la información entre un modelo candidato ajustado y el verdadero modelo (Villa, 2011). En el primer caso, dando un valor 5186.15 , y en el segundo caso 5050.30 los cuales fueron los valores más ajustados respecto a otros modelos analizados.

La lectura de las categorías se realiza a partir de los valores $d x / d y$, los cuales representan el cambio en la probabilidad de resultado $=1$ asociado con un aumento de unidad en las variables independientes correspondientes, lo cual se interpreta como la afectación (negativa o positiva) en la probabilidad de que ocurra un evento, en este caso la confianza en las autoridades, tomado como referencia una variable explicativa y manteniendo constantes las demás.

El modelo para cada variable arroja los valores $\mathrm{dx} / \mathrm{dy}$ asociados a cada una de las categorías de la variable con referencia a la primera categoría, o categoría de referencia. De esta manera, si tomamos como ejemplo la variable beca, se tienen dos posibilidades: tiene o no tiene, por tanto, el modelo toma como referencia la primera categoría y arroja un cambio marginal (positivo o negativo) en la segunda.

La institución educativa en la que estudia el alumno puede tener un efecto positivo o negativo, dependiendo a cuál se pertenezca. Según lo estimado, las variables que resultaron estadísticamente significativas son CONALEP y CETIS, las cuales afectan de manera negativa la confianza de un estudiante en sus autoridades, y las preparatorias abiertas y privadas, que tienen un efecto positivo en la variable dependiente.

En lo que se refiere al tipo de bachillerato, tanto asistir a uno de tipo tecnológico como a uno con carrera técnica, tienen un efecto positivo en la confianza de un alumno en sus autoridades (tomando como referencia al bachillerato general), siendo el último el que reporta un cambio marginal más importante. Respecto al sostenimiento, las escuelas de carácter privado manifiestan un efecto positivo respecto a la confianza en el gobierno de la escuela. 
En cuanto a la categoría de con quién vivía el estudiante al momento de estudiar la EMS, cuando se vive con al menos alguno de sus padres, ya sea madre o padre, se tiene un efecto negativo y estadísticamente significativo, en la confianza que un alumno pudiera tener en las autoridades de su escuela. Asimismo, si cuando se presenta un problema, el estudiante recurre primero a algún familiar o amigo cercano, se afecta de manera negativa la confianza hacia la autoridad escolar.

La percepción que el alumno entrevistado tiene sobre su promedio tuvo una influencia en la confianza que este tenía sobre las autoridades escolares. Los casos significativos son cuando esta percepción del promedio es regular o alta, ya que estas tienen un efecto negativo en la confianza que un alumno pueda tener en la escuela y sus representantes; sin embargo, aunque no es estadísticamente significativo, tener un promedio muy bajo tiene un efecto positivo.

El tema económico es un tópico relevante en el ámbito de la calidad educativa. Según lo estimado en este modelo, tener problemas económicos y ser poseedor de una beca durante los estudios en la EMS fortalece la relación entre los alumnos y las autoridades. Asimismo, el trabajar mientras se estudia, también lo hace de manera positiva y estadísticamente significativa.

Cuando a un alumno le disgusta estudiar y se ve forzado a hacerlo, tiene un efecto negativo en la confianza hacia las autoridades escolares; también el sentirse discriminado por su forma de vestir o pensar genera un distanciamiento con el gobierno de la escuela. Sin embargo, si el alumno tiene problemas con materias reprobadas, se aumenta el acercamiento del alumno a la autoridad de la escuela.

El tema aspiracional (cuál es el máximo nivel de estudios al que el alumno desea acceder) tiene un efecto positivo y significativo respecto a la confianza que manifiesta con sus autoridades escolares al momento de estudiar la EMS, de manera que, entre más alto sea el grado escolar al que se desea llegar, más acercamiento existe.

El uso de alcohol y/o tabaco durante la EMS genera un distanciamiento entre las autoridades escolares y sus alumnos. Sin embargo, aunque no sea estadísticamente significativo, el consumo de drogas marca un efecto positivo en la confianza de los alumnos hacia sus autoridades.

En cuanto a la asistencia escolar, hay una marcada tendencia de que entre más se asiste a la escuela menos confianza se tiene en las autoridades. El tema de la edad es un aspecto muy importante según la literatura (Pérez, 2017), pero para las estimaciones del modelo, dista mucho de ser estadísticamente significativo, razón por lo cual no se puede realizar una inferencia causal.

La primera hipótesis de este trabajo se cumple de manera parcial, ya que si bien los factores identificados como contextuales o de gestión escolar tienen un mayor peso en la decisión de abandono - además de ser en su mayoría estadísticamente significativos-, aquellos reconocidos como individuales o de composición escolar son importantes si se quiere estudiar dema-

Diólo pos sobre Educación año 10 | número 19 | julio-diciembre 2019 | ISSN 2007-2171 
nera integral la generación de confianza de las autoridades escolares en los estudiantes dentro de la EMS.

\section{Propensión marginal a la confianza}

Una vez que ya ha sido ajustado el modelo logit, pueden obtenerse las probabilidades de un evento positivo (confianza en las autoridades escolares) predichas para cada unidad de observación de la siguiente manera:

$$
\operatorname{Pr}(y j=1)=F(x j b) \quad \text { Ecuación } 6
$$

En la cual se calcula la combinación linear de $\left(x_{j} b\right)$ donde $x_{j}$ son las variables independientes en la j-ésima observación y $b$ es el vector parámetro estimado. Es así que la función de distribución acumulativa indexada en este valor es la probabilidad de un resultado positivo.

Con base en los resultados del modelo se estimaron las probabilidades por cada unidad de observación, que en este caso sería la propensión marginal de cada alumno a confiar en las autoridades, donde la probabilidad de un evento A (confiar en las autoridades) es un número real en el intervalo $[0,1]$ que se denota por $\mathrm{P}(\mathrm{A})$ y representa la medida de la frecuencia con la que se observa la ocurrencia de este evento.

Cuadro 1. Confianza de los alumnos en las autoridades

\section{Propensión marginal}

\begin{tabular}{|c|c|c|c|}
\hline Media & Desviación estándar & Mínimo & Máximo \\
\hline 0.6889 & 0.2078 & 0.0531 & 0.9775 \\
\hline
\end{tabular}

Fuente: Elaboración propia con información de la ENDEMS (2012)

Se realizó una prueba de diferencia de medias en donde se muestra que la distribución de los datos es normal y que la diferencia de medias entre abandonantes y no abandonantes es distinta de cero, por lo cual las estimaciones son estadísticamente significativas. A continuación, se muestran algunos estadísticos descriptivos de los resultados.

Cuadro 2. Confianza de los alumnos en las autoridades: abandonantes/ no abandonantes

\section{Propensión marginal}

\begin{tabular}{|l|c|c|c|}
\hline & Media & Error estándar & Desviación estándar \\
\hline Abandonantes & 0.6136 & 0.0059 & 0.2124 \\
\hline No abandonantes & 0.7133 & 0.0032 & 0.2003 \\
\hline
\end{tabular}

Fuente: Elaboración propia con datos de la ENDEMS (2012) 
Gráfica 1. Propensión marginal a confiar en las autoridades: abandonantes/ no abandonantes

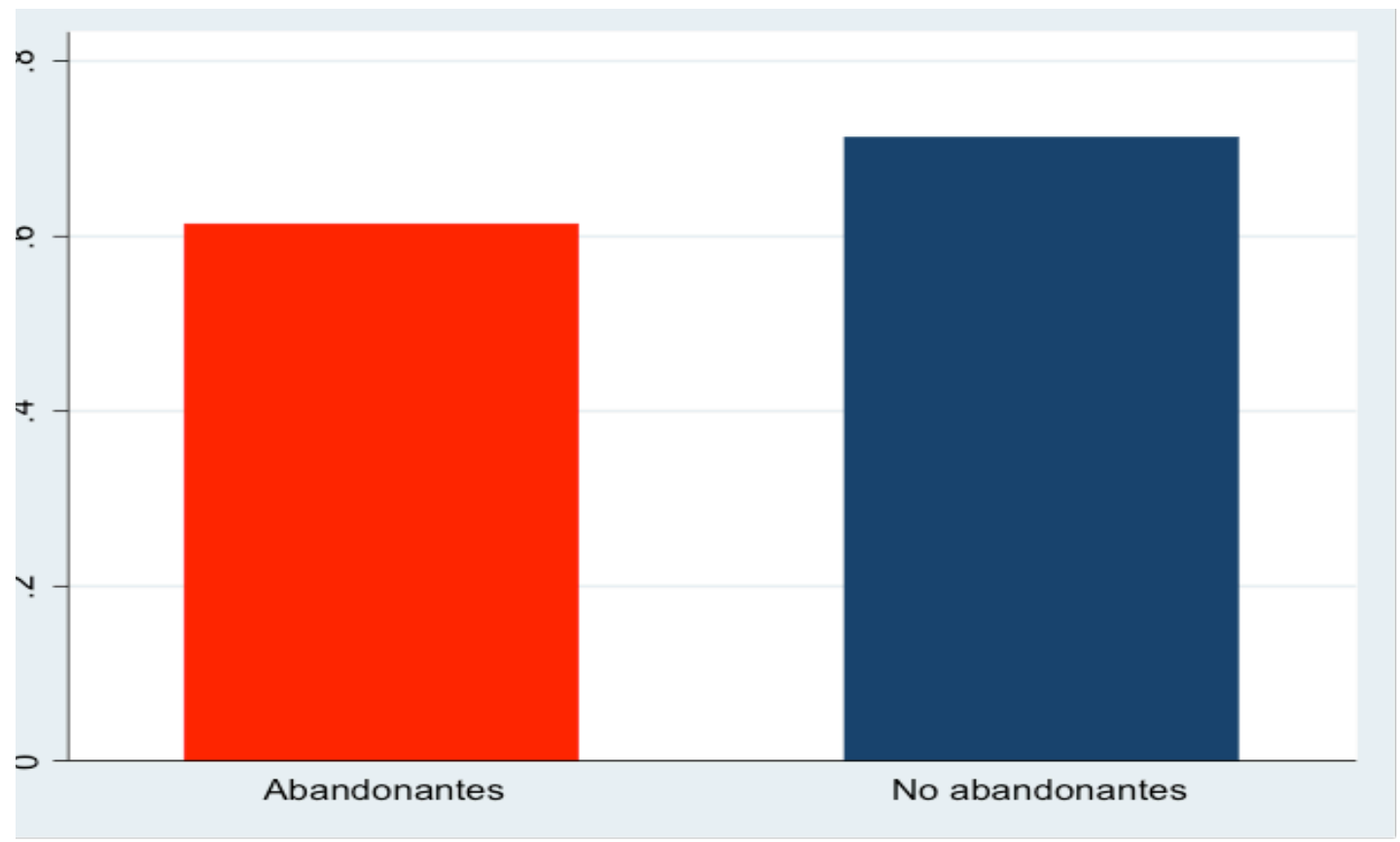

La segunda hipótesis de este trabajo se cumple ya que, como se puede observar, existe una diferencia de medias de 0.1003 entre los abandonantes y no abandonantes, lo cual se interpreta que, en promedio, los alumnos que no abandonaron sus estudios tuvieron una propensión marginal a confiar en las autoridades $10 \%$ más alta que aquellos que dejaron sus estudios de EMS incompletos.

La importancia de este hecho es que, a pesar de que la confianza en las autoridades es un factor determinante para la calidad educativa y la permanencia escolar de los alumnos en la EMS (ENDEMS 2012), no existe una forma estandarizada de cuantificarla y que explique cuáles son los factores que influyen para que un alumno confíe o no en la autoridad escolar.

Los resultados de este trabajo reflejan la importancia de tomar en consideración las relaciones intraescolares al momento de diseñar políticas públicas que busquen mejorar la calidad educativa y prevenir el abandono escolar. Si bien es cierto que esta investigación tiene la limitante de usar una fuente de información de casi siete años de antigüedad, es porque desde 2012 no se ha levantado una encuesta similar. En las bases de datos existentes, como la estadística 911, se toma en cuenta a los alumnos como un agregado, pero no se enfocan en las características individuales de estos, además de que las relaciones intraescolares, entre ellas la confianza, son un tema olvidado por este tipo de registros.

Es trascendental que se estandarice una medición de la confianza de los alumnos hacia sus autoridades, ya sea como una inclusión en la estadística 911.7 u otra fuente de información 
representativa de nivel nacional. Además, es importante que cualquier política pública enfocada la mejora de la calidad educativa tenga en consideración este factor, de forma que se cree una estrategia integral que tome en cuenta, tanto a la gestión escolar como a la composición estudiantil.

No se puede abordar este problema de manera aislada, porque una mejora en la calidad educativa se da por la integración de ambos factores, individuales y contextuales. Las relaciones intraescolares deben ser un elemento clave en el estudio ex post y ex ante de la educación, no solo en la EMS, sino en todos los niveles y los vínculos entre autoridades escolares y alumnos debe de ser eje central de este análisis.

\section{Referencias}

Ander-Egg, E. (1995). Técnicas de investigación social. Buenos Aires: Editorial Lumen.

Anderson, H. y T. Black (1999). Análisis multivariante. Nueva Jersey: Prentice Hall.

Ansell, B. W. y D. Samuels (2014). Inequality and Democratization: An Elite-Competition Approach. Cambridge: Cambridge University Press.

Arias, F. (2007). Metodología de la investigación en contabilidad, economía, administración, psicología, trabajo social y educación. México: Trillas

Aristimuño, A. (2009). "El abandono de los estudios del nivel medio en Uruguay: un problema complejo y persistente". Revista Iberoamericana sobre Eficiencia Calidad y Cambio en la Educación, Número Monográfico: Abandono y Deserción en la Educación Iberoamericana, 7(4).

Banco Mundial (abril 2017). Mejorar la flexibilidad, calidad y equidad de la educación media superior en México. Proyectos y operaciones. http://www.bancomundial.org/es/results/2017/04/10/ improving-flexibility-quality-equity-upper-secondary-education-mexico

Barrientos Noriega, A. I. y E. Taracena Ruiz (2008). "La participación y estilos de gestión escolar de directores de secundaria. Un estudio de Caso". Revista Mexicana de Investigación Educativa, 13(36), 113-114.

Batallán, G. (2003). “El poder y la autoridad en la escuela. La conflictividad de las relaciones escolares desde la perspectiva de los docentes de infancia". Revista Mexicana de Investigación Educativa, 8(19).

Bazúa, F. (2010). Estado, gobierno y políticas públicas. Elementos para un marco conceptual básico. México: Documento de Trabajo.

Besley, T. (2013). "What's the Good of the Market? An Essay on Michael Sandel's What Money Can't Buy". Journal of Economic Literature, 51(2), 478- 495.

Borooah, V. (2001). Logit \& Probit. Ordered and multinomial models. Arizona: Sage publications. Bollen, K. A. (1989). Structural Equations with Latent Variables. Nueva York: Willey \& Sons. 
Bryk, A. S. y B. Schneider (2003). "La confianza en las escuelas: Un recurso clave para la reforma escolar". Educational Leadership, 60(6).

Cervini, R. (2003). "Relaciones entre composición estudiantil, proceso escolar y el logro en matemáticas en la educación secundaria en Argentina". Revista Electrónica de investigación educativa, 5(1). http://www.scielo.org.mx/scielo.php?script=sci arttext\&pid $=$ S1607-40412003000100004

Cornejo, R. y J. Redondo (2001). El clima escolar percibido por los alumnos de enseñanza media. Una investigación en algunos liceos de la Región metropolitana. Viña del mar: Última década n¹5, cidpa,

et al. (2010). "Confianza: un valor necesario y ausente en la educación chilena". Perfiles Educativos, 32(129).

Dixit, A. y J. Londregan (1996). "The Determinants of Success of Special Interests in Redistributive Politics". Journal of Politics, 58(4), 1132-55.

Espíndola, L. (2002). “La deserción escolar en América Latina: un tema prioritario para la agenda regional". Revista lberoamericana de Educación, 30, 30-62.

INEE (2015). "Evaluación de la política educativa". Gaceta de la Política Nacional de Evaluación Educativa en México. México: INEE.

(2007). Factores escolares y aprendizaje en México, el caso de la educación básica. México: INEE.

(2014). El derecho a una educación de calidad: Informe 2014. México: INEE. (2017). Panorama educativo 2016. México: INEE.

Kreft, I. (1993). “Using multilevel analysis to assess school effectiveness: A study of Dutch secondary education". Sociology of Education, 66(2), 104-129.

Malhortra, N. (2004). Investigación de Mercados, un enfoque aplicado. México: Prentice Hall

Mejía-Bustamante (2015). "La confianza en el aula como eje transformador del mundo escolar". Sophia. Revista de investigaciones en educación, 11(2).

Miranda, F., et al. (2017). Directrices para mejorar la permanencia escolar en la educación media superior. México: INEE.

(2017). Análisis de atención de la política de atención al abandono escolar en la educación media superior. La educación obligatoria en México informe 2017. México: INEE.

Muñoz, V. (2007). "El Derecho a la Educación en el mundo: miradas a las metas de Educación para Todos". Asamblea General de la Campaña Mundial para la Educación. Sao Paulo, 24 de enero. http://www.incidenciaeducacion.org.mx/

Montesinos-López, A. (2011). Estudio del AIC y BIC en la selección de modelos de vida con datos censurados. México: Centro de Investigación en Matemáticas.

Peña, D. (2002). Análisis de datos multivariantes. México: Mc Graw Hill.

Pérez, A. (2000). Jóvenes e instituciones en México, actores, políticas y programas. México: SEP-IMJ. 
et al. (2017). "Cómo hacer que los jóvenes se queden en las aulas". Gaceta de la Política Nacional de Evaluación Educativa. México: INEE.

Schmelkes, S. (2008). Entre el desencanto y la fe por la educación y las cuestiones sociales. México: CREFAL.

Solís, P., E. Blanco et al. (2014) Caminos desiguales: Trayectorias educativas y laborales de los jóvenes en la Ciudad de México. México: El Colegio de México.

Székely, M. (2015). "Estudio, resultados y recomendaciones de la investigación y evaluación educativa en el eje de prevención y atención al abandono en EMS" (documento interno). México: FLACSO. 